\title{
Comparison of High Flow Nasal Cannula Oxygen and Conventional Oxygen Therapy on Early Effect Hemodynamic Indicators in Post - Upper Abdominal Surgery Patients
}

\author{
Luki Sumaratih ${ }^{1}$, Rudyanto Soedono ${ }^{2}$, Dita Aditianingsih ${ }^{2}$ \\ ${ }^{1}$ Trainee of Intensive Care Program, Universitas Indonesia-RSCM, Jakarta-Indonesia \\ ${ }^{2}$ Staff Lecturer, Department of Anesthesia and Intensive Care, Universitas Indonesia-RSCM, Jakarta-Indonesia \\ *Corresponding Author E-mail: lukicafk@gmail.com
}

Received : August $17^{\text {th }} 2019$

Accepted : October $28^{\text {th }} 2019$

\begin{abstract}
Background

The effect of high-flow nasal cannula (HFNC) on hemodynamic data of the patients is still unknown. This was a pilot study to evaluate the effect of HFNC and conventional oxygen therapy (COT) on post-upper abdominal surgery.
\end{abstract}

Methods

This was an open-label randomized controlled trial (RCT) at tertiary hospital between February-June 2019. Inclusion criteria were ASA III, aged 18-65 years, BMI 18,5-29 kg/m². Exclusion criteria were patients with unstable hemodynamic with vasopressors, general oedema, and uncooperative. Thirty patients were recruited and divided into HFNC group $(n=15)$ and COT group $(n=15)$. Hemodynamic parameters were recorded using the bedside monitor (heart rate, respiratory rate, and mean arterial pressure) as well as the electrical cardiometry using ICON $®$ measurements (stroke volume index, cardiac index and systemic vascular resistance index); laboratory parameters were $\mathrm{ScVO}_{2}$ and lactate serum. Data were collected at 0,30 and 60 minutes postextubation.

Results

The General Linear Model (GLM) tests on clinical parameters (HR, RR and MAP) were $P=0.20 ; P$ $=0.72 ; P=0.18, \mathrm{ICON} \circledast$ parameters $(\mathrm{SVI}, \mathrm{CI}$ and SVRI) were $P=0.02 ; P=0.64 ; P=0.64$ meanwhile ScVO2 and lactate were $P=0.35$ and $P=0.22$. Despite those results, at some period of measurements there were a tendency of a better outcomes of most parameters for the HFNC group.

Conclusion

According to the results, the only statistically significant outcome was SVI $\quad(P=0.02)$ after the analysis between those two groups. However, the HFNC group showed a clinically improvement of the other outcomes among post-upper abdominal surgery patients.

Keywords : High flow nasal cannula, conventional oxygen therapy, haemodynamic, abdominal surgery

\section{Introduction}

The first hour post-extubation is a critical time for the ICU patients as they convert from mechanical ventilation into their physiological respiration. Switching from mechanical to spontaneous ventilation increased work of breathing that can decrease left ventricular 
ISSN 25980580

performance and unmask latent left ventricular dysfunction by increasing preload and afterload. It was reported that 12 to $14 \%$ patients who undergo planned extubation would require reintubation within the first 24 hours. [1,2] Postoperative pulmonary complication is one of the most common reintubation complications following abdominal surgery, the incidence varied from 2 to 19\%. [3, 4]. The other causes are sepsis, surgical complication, neurological impairment and acute coronary syndrome. [2]

For years supplemental oxygen administration was administered by conventional devices, such as nasal prongs, nose masks and face masks. However, this method has several limitations, including insufficient oxygen flow to correct hypoxemia for patients with high demand. Recently, high flow nasal cannula oxygen therapy (HFNC) is one of the alternative which works with an air oxygen blender allowing from $21 \%$ to $100 \% \mathrm{FiO} 2$ and generates up to $60 \mathrm{~L} / \mathrm{min}$ flow rates. [5, 6] High flow therapy can minimize the inspiratory resistance that causes decreasing resistive work of breathing and minimal elevation in intrathoracic pressure that contributes to hemodynamic state [6].

This was a pilot study to evaluate the early effects of HFNC and conventional oxygen therapy (COT) on post-upper abdominal surgery in adult patients on macrocirculation parameters which were stroke volume index (SVI), systemic vascular resistance index (SVRI), cardiac index (CI) and microcirculation parameters $\left(\mathrm{ScVO}_{2}\right.$, serum lactate).

\section{Materials and Methods}

This was an open-label randomized controlled trial (RCT) at tertiary hospital between February-June 2019. Inclusion criteria were physical status American Society of Anaesthesiologists (ASA) III, aged 18-65 year, scheduled for upper-abdominal surgery and Body Mass Index (BMI) of 18.5-29 $\mathrm{kg} / \mathrm{m}^{2}$. Exclusion criteria were patients with unstable hemodynamic with vasopressors, general oedema, and uncooperative. Thirty-two patients were recruited and divided into Group HFNC $(n=16)$ and Group COT $(n=16)$.

During intra-anaesthesia procedures prior to the surgery, general anaesthesia combined with epidural regional anaesthesia. Central venous catheter was inserted with the projection of the tip of catheter located within the superior vein cava (SVC), above its junction with the right atrium. Mechanical ventilation using lung protective strategy, volume controlled, tidal volume of $6-8 \mathrm{ml} / \mathrm{KgBW}$, respiratory rates of $12-14 \mathrm{x} /$ minutes, positive end expiratory pressure 
ISSN 25980580

(PEEP) of $5-10 \mathrm{cmH}_{2} 0, \mathrm{FIO}_{2}$ of $30-50 \%$ with oxygen saturation $\left(\mathrm{SaO}_{2}\right)$ target of $>92 \%$, and end- tidal $\mathrm{CO}_{2}\left(\mathrm{EtCO}_{2}\right)$ of $30-40 \mathrm{mmHg}$. After transported to intensive care unit (ICU) a midazolam sedation was administered at $0.015 \mathrm{mg} / \mathrm{KgBW}$ dosage with the target of Ramsay sedation score (RSS) of 2. Multi-modal analgesia with non-steroidal anti-inflammatory drugs (NSAIDs) and epidural were administered with the visual analogue scale (VAS) target of $<3$. For intervention group, the standard settings for $\mathrm{HFNC}$ are $34^{\circ} \mathrm{C}, 35 \mathrm{~L} / \mathrm{min}$ flow, and $21 \%$ $\mathrm{FiO}_{2}$. If needed, we titrate the $\mathrm{FiO}_{2}$ until $\mathrm{SpO}_{2}$ target $>92 \%$ was reached. The maximum concentration of $\mathrm{FiO}_{2}$ to be used for the therapy was 35\%. For the control group, we administered routine nasal cannula oxygen therapy $3-4 \mathrm{~L} / \mathrm{min}$ and $\mathrm{FIO}_{2} 21 \%$.

Hemodynamic parameters were recorded by using the bedside monitor (heart rate, respiratory rate, and mean arterial pressure) and electrical cardiometry by using ICON® measurements (stroke volume index, cardiac index and systemic vascular resistance index); meanwhile, laboratory parameters were $\mathrm{ScvO}_{2}$ and lactate serum. Data were collected at 0,30 and 60-minutes post-extubation.

After the surgical procedures were finished, the patients were transported to Intensive Care Unit (ICU) after declared as "safe from the post-surgical recovery room. Routine clinical and laboratory examinations were performed prior to the extubation process. Once the patient is declared safe for extubation, the extubation process took place and the data were collected 30 minutes after the process. We used a calibrated bed-side monitor with probes to collect data for the clinical outcomes. The data was recorded three times with the spare of 1 minute for each measurement. For the cardiometry parameter we used ICON ® by Osypka with four probes located in jugular vein and the chest. We also collected three data for each measurement and calculate the mean value for each. For the laboratory outcomes, the blood sample was taken from central venous catheter and arterial line. The samples were then analysed by the calibrated pHOx plus by Nova biomedical located inside the ICU and drops of each sample were also analysed for serum lactate measurement using the device from Nova biomedical as well.

The statistical analysis was conducted by using General Linear Model (GLM) study with SPSS 23 program. The statistical power used was 95\% CI with $p<0.05$ determines the significance.

\section{Results}


This study included 30 subjects divided into 2 groups which were Group COT and HFNC. The characteristics were shown in Table 1. The outcomes were shown as mean values with three courses measurements that categorized into clinical, ICON®, and laboratory parameters.

Table 1. Baseline characteristics of both groups

\begin{tabular}{lcc}
\hline Characteristics & COT Group $(\mathrm{n}=15)$ & HFNC Group $(\mathrm{n}=15)$ \\
\hline Age & $45.33 \pm 13.83$ & $47.93 \pm 11.49$ \\
Sex Male. $(\%)$ & $5(33 \%)$ & $5(33 \%)$ \\
\multicolumn{1}{c}{ Female. $(\%)$} & $10(67 \%)$ & $10(67 \%)$ \\
BMI $\left(\mathrm{kg} / \mathrm{m}^{2}\right)$ & $21.65 \pm 1.87$ & $22.91 \pm 3.51$ \\
Hemoglobin $(\mathrm{g} / \mathrm{dL})$ & $11.77 \pm 2.44$ & $10.65 \pm 1.66$ \\
Duration of Operation & $226.86 \pm 75.95$ & $240(120-675)$ \\
Blood Loss & $200(20-1500)$ & $600(100-2500)$ \\
Types of Surgery & $0(0 \%)$ & $1(6.67 \%)$ \\
Hepatobiliary Surgery & $13(86.67 \%)$ & $9(60 \%)$ \\
Gastrointestinal Surgery & $2(13.33 \%)$ & $5(33.33 \%)$ \\
Obstetric Surgery & & \\
\hline
\end{tabular}

We excluded two patients due to the occurrence of reintubation and prolonged time of intubation.

We analysed heart rate, respiratory rate, and mean arterial pressure for the clinical outcomes at time 0 (baseline) followed by $30^{\text {th }}$ and $60^{\text {th }}$ minute measurements. From the results, it was shown that the HFNC Group had lower means for heart rate compared to the COT group, although the $p$ value showed that it was not statistically significant $(p=0.205)$. The similar 
result was also found in respiratory rate measurements $(p=0.729)$. The result for MAP measurement showed almost similar value for each group $(p=0.184)$ as seen on table 2 . There was one patient who complained the dryness on nose tip due to warm temperature of air flow $\left(34^{\circ} \mathrm{C}\right)$, we change the temperature into $28^{\circ} \mathrm{C}$, and the problem was resolved.

For ICON ${ }^{\circledR}$ external cardiometry parameters, we analysed the SVI, SVRI, and CI. The SVI was the only parameter that showed significant difference between each group. As shown in figure 1, both groups showed an increase of mean SVI after the first 30 minutes of oxygen therapy, for the next 30 minutes, the HFNC group differed from COT group by continuously increasing while COT group decreased. For CI parameter, as seen in table 2, the HFNC group showed a constant increase within each course of measurement while the COT group showed more fluctuated result. As for the SVRI outcomes, the HFNC group showed lesser value for each course of measurement compared to the COT group.

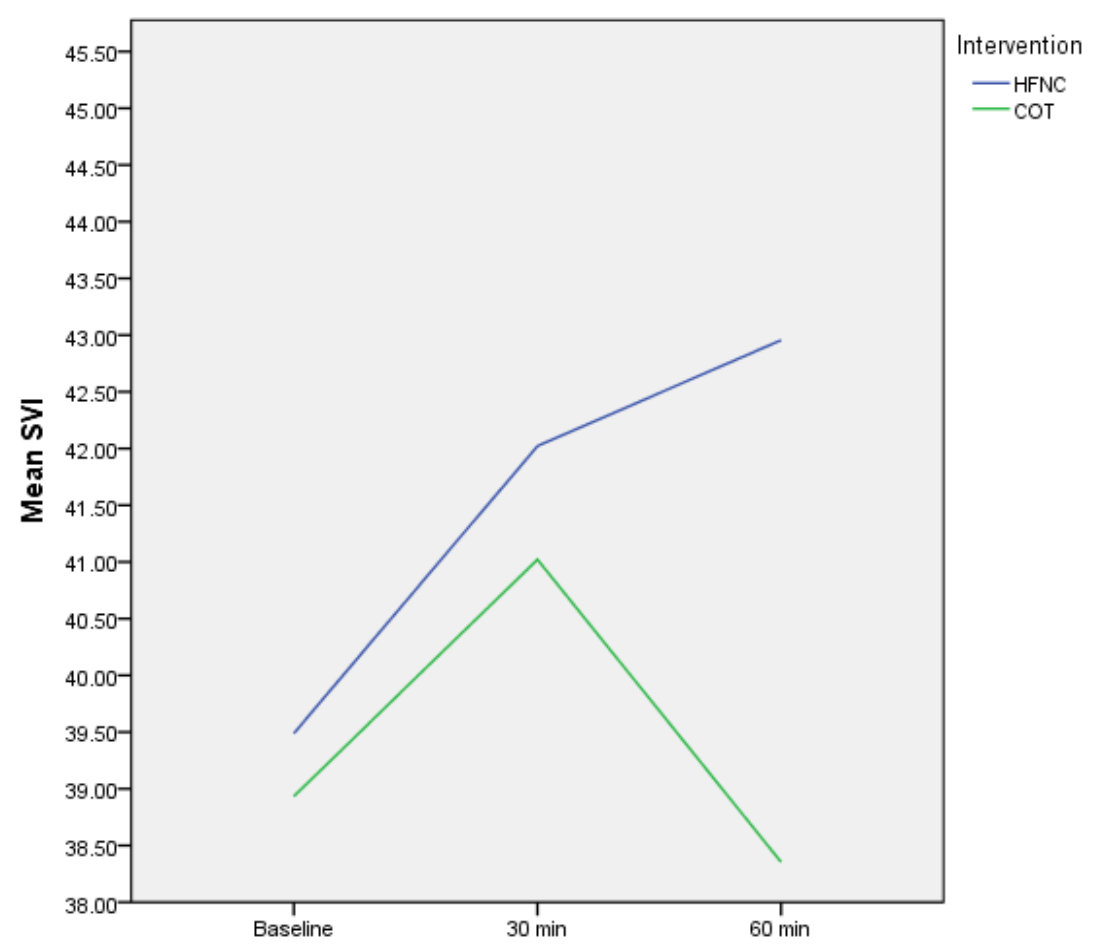

Figure 1. Mean SVI comparison between the two groups

The laboratory outcomes were $\mathrm{ScvO}_{2}$ of mixed vein (MV) and serum lactate. Both parameters showed almost similar outcomes between the two groups, but the constant reduction of serum lactate value was achieved in HFNC group compared to the COT group. 
ISSN 25980580

Table 2. Mean values comparison between the two groups through three courses of measurements

\begin{tabular}{|c|c|c|c|c|}
\hline $\begin{array}{l}\text { Outcome } \\
\text { Parameters }\end{array}$ & $\begin{array}{l}\text { Baseline } \\
\text { (COT vs HFNC) }\end{array}$ & $\begin{array}{ll}\text { 30-minutes After } & \\
\text { Extubation } & \\
(\text { COT vs HFNC) } & \end{array}$ & $\begin{array}{l}\text { 60-minutes After } \\
\text { Extubation } \\
(\text { COT vs HFNC) }\end{array}$ & $\begin{array}{l}p \\
\text { value } \\
\text { by } \\
\text { GLM }\end{array}$ \\
\hline $\mathrm{RR}(\mathrm{x} / \mathrm{min})$ & $\begin{array}{l}18.93 \pm 4.51 \mathrm{vs} \\
16.53 \pm 3.04\end{array}$ & $\begin{array}{l}19.46 \pm 3.37 \mathrm{vs} \\
16.33 \pm 3.39\end{array}$ & $\begin{array}{l}18.00 \pm 4.29 \mathrm{vs} \\
15.60 \pm 4.25\end{array}$ & 0.729 \\
\hline $\mathrm{HR}(\mathrm{x} / \mathrm{min})$ & $\begin{array}{l}98.93 \pm 18.62 \text { vs } \\
88.73 \pm 16.82\end{array}$ & $\begin{array}{l}96.46 \pm 21.88 \text { vs } \\
93.2 \pm 13.15\end{array}$ & $\begin{array}{l}97.20 \pm 20.6 \mathrm{vs} \\
93.6 \pm 12.89\end{array}$ & 0.205 \\
\hline $\begin{array}{l}\text { MAP } \\
(\mathrm{mmHg})\end{array}$ & $\begin{array}{l}86.62 \pm 8.86 \mathrm{vs} \\
87.95 \pm 9.14\end{array}$ & $\begin{array}{l}87.73 \pm 9.63 \mathrm{vs} \\
88.80 \pm 9.44\end{array}$ & $\begin{array}{l}86.73 \pm 8.94 \mathrm{vs} \\
85.08 \pm 8.57\end{array}$ & 0.184 \\
\hline $\mathrm{SVI}\left(\mathrm{ml} / \mathrm{m}^{2}\right)$ & $\begin{array}{l}38.93 \pm 8.96 \mathrm{vs} \\
39.48 \pm 11.77\end{array}$ & $\begin{array}{l}41.02 \pm 9.83 \mathrm{vs} \\
42.02 \pm 9.16\end{array}$ & $\begin{array}{l}38.35 \pm 7.75 \mathrm{vs} \\
42.95 \pm 9.07\end{array}$ & 0.021 \\
\hline $\begin{array}{l}\text { SVRI } \\
\left(\mathrm{d} / \mathrm{s} / \mathrm{m}^{2}\right)\end{array}$ & $\begin{array}{l}2042.48 \pm 584.65 \mathrm{vs} \\
1988.64 \pm 592.16\end{array}$ & $\begin{array}{l}1865.30 \pm 617.24 \mathrm{vs} \\
1746.6 \pm 337.09\end{array}$ & $\begin{array}{l}1926.91 \pm 625.01 \mathrm{vs} \\
1667.42 \pm 364.95\end{array}$ & 0.131 \\
\hline $\begin{array}{l}\text { CI } \\
\left(\mathrm{L} / \mathrm{min} / \mathrm{m}^{2}\right)\end{array}$ & $\begin{array}{l}3.56 \pm 0.95 \mathrm{vs} \\
3.53 \pm 1.11\end{array}$ & $\begin{array}{l}3.88 \pm 1.01 \mathrm{vs} \\
3.91 \pm 0.77\end{array}$ & $\begin{array}{l}3.66 \pm 0.90 \mathrm{vs} \\
4.04 \pm 0.73\end{array}$ & 0.064 \\
\hline MV (\%) & $\begin{array}{l}78.96 \pm 9.26 \mathrm{vs} \\
81.26 \pm 6.56\end{array}$ & $\begin{array}{l}80.05 \pm 8.36 \mathrm{vs} \\
83.10 \pm 5.77\end{array}$ & $\begin{array}{l}80.16 \pm 6.50 \mathrm{vs} \\
80.43 \pm 6.22\end{array}$ & 0.355 \\
\hline $\mathrm{Lac}(\mathrm{g} / \mathrm{dL})$ & $\begin{array}{l}1.94 \pm 0.92 \mathrm{vs} \\
1.97 \pm 0.76\end{array}$ & $\begin{array}{l}1.95 \pm 1.15 \mathrm{vs} \\
1.60 \pm 0.78\end{array}$ & $\begin{array}{l}1.95 \pm 1.16 \mathrm{vs} \\
1.48 \pm 0.75\end{array}$ & 0.229 \\
\hline
\end{tabular}

Note : RR - respiratory rate; $H R$ - heart rate; $M A P$ - mean arterial pressure; $S V I$ - stroke volume index; SVRI - stroke volume variation index, CI - cardiac index; $M V$ - mixed vein; Lac-lactate

\section{Discussion}

This pilot study was aimed to evaluate the haemodynamic effect of HFNC and COT therapy on post-upper abdominal surgery patients. This study would like to identify the 
ISSN 25980580

difference between two groups through course of time postoperatively, they were divided into three major surgeries: hepatobiliary surgery (3.3\%), gastro-intestinal surgery $(73.33 \%)$ and obstetric surgery $(23.33 \%)$. This was because this study expected to obtain similar outcomes on respiratory and haemodynamic effects of these procedures. To reduce bias this studied included duration of operation, haemoglobin level, sum of blood loss, and fluid resuscitation into the analysis.

This study showed no significant difference for all parameters, except for the SVI. For clinical outcomes, both RR and HR were lower in Group HFNC compared to Group COT. The decrease of RR could be used as an indication for work of breathing, this finding should be concordance with the decrease of heart rate as it also indicates the reduction of sympathetic activity, thus, lowering the workload of the heart. [7] The normal value of both RR and HR comes in range which are 12 to 20 and 80 to $100 \mathrm{x} / \mathrm{min}$ respectively. We can see on the table that there was a constant reduction of mean RR for HFNC group compared to COT group. For the first 30 minutes, there was an increase of RR in COT group although it was then reduced to normal by the compensatory respiratory mechanism. On the other side, in the HFNC group, the RR was constantly reduced and maintained within the normal range. Similar finding was also found in meta-analysis by Ni et al (2017) who concluded that HFNC reduce reintubation by decreasing respiratory rate. [1]

The mechanism of how HFNC decreased respiratory rate and work of breathing can be explained as the followings. First of all, as mechanical ventilation will leads to atelectasis even after extubation. [8, 9] A PEEP $(2-5 \mathrm{cmH} 2 \mathrm{O})$ generated by high flow can lead to continuous alveolar recruitment, reduction of airway collapse and improvement of the ventilationperfusion mismatch [10-12]. Hence larger end expiratory lung volume was observed in patients with HFNC than COT.[13] In addition, the heated humidification closely to physiological conditions preserves the mucosal function and facilitates secretion clearance, thereby further decreasing the risk of atelectasis and improving the oxygenation.[14] Second, adequate minute ventilation and sufficient oxygenation guaranteed by HFNC via delivering a continuous high flow of oxygen accompanied with a higher tidal volume and improved inspiratory flow dynamics. $[15,16]$ For HR parameter, the reduction was relatively the same for both groups. Although the HFNC group showed lower mean HR compared to the COT group as this is concordance with the RR reduction. 
ISSN 25980580

For electrical cardiometry parameters, ICON ${ }^{\circledR}$ works by calculating the cardiac parameters based on the patient's clinical data. The normal value of each measurements will be shown as range from low to high. The number is determined by calculating the patient's BMI, age, haemoglobin level, and haemodynamic status (HR, RR, MAP, SpO2). This study generated that HFNC group had significant higher mean SVI compared to COT group ( $p=$ 0.02). This condition was due to preserved acute hypoxia stroke volume despite the higher HR, indicating enhanced venous return. Acute hypoxia stroke volume was related to the vascular effect of hypoxia-induced sympathoactivation, to the higher ventilation and/or the accelerated HR which increases the occurrence of negative pressure in the right atrium. In contrast a reduced SV is a universal finding in chronic hypoxia. [17] Even the SVI was higher in HFNC group, it was still within normal values based on BMI. Secondly, this higher finding of mean SVI might be due to decrease afterload since the determinants of SV were myocardial contractility, left ventricular preload and afterload. [18, 19]

Group HFNC had higher mean value of ScvO2, which still within the normal range. This indicated that group HFNC had normal oxygen consumption. Low $\mathrm{ScvO}_{2}$ showed inadequate stroke volume with exaggerate oxygen extraction as seen in acute respiratory failure, low haemoglobin level or low $\mathrm{PaO}$. Meanwhile, high $\mathrm{ScvO}_{2}$ indicated high oxygen delivery which exceed tissue needs, decreased oxygen consumption due to mitochondrial dysfunction or arterial venous shunt.[20-22] All subjects underwent a lower abdominal surgery which might contribute to lower $\mathrm{ScvO}_{2}$ due to haemorrhage, pain, increased metabolic demand, hypovolemia and hypoxia. As shown in Table 2 there was no significant difference between two groups.

This study also found that group HFNC had lower serum lactate level which indicated better oxygen delivery through lesser anaerobic metabolism. A serum lactate can reveal certain condition like oxygen deficit within the cellular level. This oxygen deficit can be caused by hypovolemia or decrease of perfusion as found in our subjects. Serum lactate also can reveal more of the body condition as it represents the work mitochondrial enzymatic system. This system is responsible for the production of lactic acid; hence, lacticaemia could be used to express adverse biochemical changes and anaerobiosis condition which also represented inadequate tissue oxygenation. [21, 23] In this study after three courses of measurements, the mean lactate level was lower in HFNC group compared to the COT group. 
There were several limitations of this study. First, this was a pilot study with no references for hemodynamic parameters for Asian population. Secondly, this study did not specify the surgery type since lower abdominal surgery had various stress response depends on the type of surgery. Therefore, further study with longer duration of observation was required to observe any significant difference. Additionally, further study might utilize invasive hemodynamic monitoring to obtain more accurate data.

\section{Conclusion}

There was no statistically significant difference for the outcomes except for the SVI parameter between the two groups. Despite the results, the HFNC group showed a better improvement for the measured parameters.

\section{Acknowledgements}

The authors would like to thank the staff in Cipto Mangunkusumo Hospital, Department of Anaesthesiology and Intensive Care, University of Indonesia, for their support of this research project, which was a part of thesis for intensive care consultant.

\section{References}

1. Ni, Y.-N., et al., Can high-flow nasal cannula reduce the rate of reintubation in adult patients after extubation? A meta-analysis. BMC Pulmmed. 2017; 17 (1): 142.

2. Thille, A.W., J.-C.M. Richard, and L. Brochard, The decision to extubate in the intensive care unit. Am J Respir Crit Care Med. 2013;187 (12): 1294-1302.

3. Canet, J., et al., Prediction of postoperative pulmonary complications in a populationbased surgical cohort. Anesthesiology. 2010; 113 (6):1338-50.

4 Fisher, B.W., S.R. Majumdar, and F.A. McAlister, Predicting pulmonary complications after nonthoracic surgery: a systematic review of blinded studies. Am J med. 2002; 112 (3): 219-225.

5. Gotera C, Lobato SD, Pinto T, Winck J. Clinical evidence on high flow oxygen therapy and active humidification in adults. Rev Port Pneumol. 2013; 19 (5): 217-27.

6. Dysart K, Miller TL, Wolfson MR, Shaffer TH. Research in high flow therapy: mechanisms of action. Respir Med. 2009 (10); 103: 1400-5. 
7. Derchak, P.A., et al., Effects of expiratory muscle work on muscle sympathetic nerve activity. J Appl Physiol. 2002; 92 (4): 1539-1552.

8. Joyce, C. and A. Williams, Kinetics of absorption atelectasis during anesthesia: a mathematical model. J Appl Physiol. 1999; 86 (4): 1116-1125.

9. Edmark, L., et al., Oxygen concentration and characteristics of progressive atelectasis formation during anaesthesia. Acta Anaesthesiol Scand. 2011; 55 (1): 75-81.

10. Corley, A., et al., Oxygen delivery through high-flow nasal cannulae increase endexpiratory lung volume and reduce respiratory rate in post-cardiac surgical patients. Br J Anaesth. 2011; 107 (6): 998-1004.

11. Parke, R.L., M.L. Eccleston, and S.P. McGuinness, The effects of flow on airway pressure during nasal high-flow oxygen therapy. Resp Care.2011; 56 (8): 1151-1155.

12. Ritchie, J., et al., Evaluation of a humidified nasal high-flow oxygen system, using oxygraphy, capnography and measurement of upper airway pressures. Anaesth Intensive Care. 2011; 39 (6): 1103-1110.

13. Riera, J., et al., Effect of high-flow nasal cannula and body position on end-expiratory lung volume: a cohort study using electrical impedance tomography. Resp Care. 2013; 58 (4): 589-596.

14. Kernick, J. and J. Magarey, What is the evidence for the use of high flow nasal cannula oxygen in adult patients admitted to critical care units? A systematic review. Aust Crit Care. 2010; 23(2): 53-70.

15. Lemiale, V., et al., The effects of a 2-h trial of high-flow oxygen by nasal cannula versus Venturi mask in immunocompromised patients with hypoxemic acute respiratory failure: a multicenter randomized trial. Crit Care. 2015; 19 (1) : 380.

16. Frizzola, M., et al., High-flow nasal cannula: impact on oxygenation and ventilation in an acute lung injury model. Pediatr pulmonol. 2011; 46: 67-74.

17. Siebenmann, C. and C. Lundby, Regulation of cardiac output in hypoxia. Scand J Med Sci Sports. 2015; 25: 53-59.

18. Lee, Q.Y., et al., Estimation of cardiac output and systemic vascular resistance using a multivariate regression model with features selected from the finger photoplethysmogram and routine cardiovascular measurements. Biomed Eng Online. 2013; 12: 19. 
19. Joshi, R., B. de Witt, and J.M. Mosier, Optimizing oxygen delivery in the critically ill: the utility of lactate and central venous oxygen saturation $(\mathrm{ScvO} 2)$ as a roadmap of resuscitation in shock. J Emerg Med. 2014; 47: 493-500.

20. Lifesciences, E., Understanding continuous mixed venous oxygen saturation (SvO2) monitoring with the Swan-Ganz oximetry TD system. 2002.

21. Vincent, J.-L. and D. De Backer, Circulatory shock. N Engl J Med. 2014; 370 (6): 583.

22. Van Beest, P., et al., Clinical review: use of venous oxygen saturations as a goal-a yet unfinished puzzle. Crit Care, 2011; 15 (5): 232.

23. Poblete, G.H., M.W. Nijsten, and J. Bakker, Lactate, in Monitoring Tissue Perfusion in Shock. 2018, Springer. 131-142. 